\title{
FIELDS WITH FEW EXTENSIONS
}

\author{
J. KNOPFMACHER AND A. M. SINCLAIR
}

Abstract. We show that a valued field $\boldsymbol{\Lambda}$ with only a finite number of nonisomorphic valued extensions is equal to the complex field $\boldsymbol{C}$ or is real closed with $C=\Lambda(\sqrt{ }(-1))$.

The Ostrowski (Gelfand-Mazur) Theorem [2, p. 131], [4, p. 260] implies that with any of the valuations $v(x)=|x|^{t}$, where $|\cdot|$ denotes the usual modulus and $0<t \leqq 1$, the real field $R$ has essentially only one proper valued field extension, the complex field $C$, and the complex field has no proper valued field extension. We investigate which valued fields $\boldsymbol{\Lambda}$ have only a finite number of nonisomorphic valued field extensions. We take all valuations to be subadditive maps into $R$, and (unless specifically stated otherwise) all isomorphisms of [valued] extensions of a [valued] field $\Lambda$ to be [isometric] $\Lambda$-algebra isomorphisms.

Before considering the case of valued fields, we consider a purely algebraic case by restricting to finite dimensional extensions. A field with no proper finite dimensional extensions is algebraically closed, since an extension of a field by a single algebraic element is a finite dimensional extension. On the other hand, it is well known that a real closed field has essentially just one proper finite extension, where a real closed field is a formally real field $\Lambda$ (that is, no sum of squares of nonzero elements in $\Lambda$ is zero) such that no proper algebraic extension of $\Lambda$ is formally real [4], [5].

1 Proposition. A field $\Lambda$ has only a finite number of finite dimensional nonisomorphic extensions if, and only if, $\Lambda$ is algebraically closed or real closed.

Proof. Suppose that there are only a finite number of finite dimensional nonisomorphic extensions of $\Lambda$. Let $\Phi$ be an extension of maximal finite degree over $\Lambda$. If $\Phi$ is not algebraically closed, then there is a prime polynomial $p$ of degree greater than 1 over $\Lambda$. Then $\Phi^{\prime}$ $=\Phi[x] /(p)$ is a proper finite extension of $\Phi$, and hence a finite extension of $\Lambda$ of degree greater than the degree of $\Phi$. Since this is impossible, $\Phi$ must be algebraically closed. If $\Phi \neq \Lambda$, the Artin-Schreier The-

Received by the editors June 9,1970 .

AMS 1969 subject classifications. Primary 1270; Secondary 1245.

Key words and phrases. Valuations, real closed fields, algebraically closed fields, real field, complex field. 
orem $[4$, p. 316] implies that $\Lambda$ is real closed (and $\Phi=\Lambda(\sqrt{ }(-1))$. The proposition follows.

Now recall that the only archimedean valuations on the complex field are of the form $v_{t}(x)=|x|^{t}$ for all $x$ in $C$ where $0<t \leqq 1$.

2 Proposition. A valued field $(\Lambda, v)$ has only a finite number of nonisomorphic valued field extensions if, and only if, $(\Lambda, v) \cong\left(\boldsymbol{C}, v_{t}\right)$ or $\Lambda$ is real closed with $\Lambda(\sqrt{ }(-1)) \cong C$ and $v$ corresponds to a restriction of $v_{t}$ for some $t(0<t \leqq 1)$.

Proof. Suppose that the second condition is satisfied: if $(\Lambda, v)$ $\cong\left(\boldsymbol{C}, v_{t}\right)$ or $\left(\boldsymbol{R}, v_{t}\right)$, then, by the Gelfand-Mazur Theorem [2, p. 127], the only valued extensions of $\Lambda$ are essentially $\left(C, v_{t}\right)$ or $\left(C, v_{t}\right)$, $\left(R, v_{t}\right)$, respectively. If $(\Lambda(\sqrt{ }(-1)), v) \cong\left(C, v_{t}\right)$ and $(\Lambda, v) \nsubseteq\left(R, v_{t}\right)$ or $\left(C, v_{t}\right)$, let $\Phi$ be a proper valued extension of $\Lambda$. Then, since the valuation on $\Phi$ is archimedean, by Ostrowski's Theorem [2, p. 131], there is an isomorphism $\theta$ from $\Phi$ onto a dense subfield of $\boldsymbol{C}$ such that $v=v_{r} \theta$ for some $r(0<r \leqq 1)$. Now $\theta$ restricted to $\Lambda$ induces an isometric isomorphism from a dense subfield of $\left(C, v_{t}\right)$ (isomorphic to $\Lambda$ and of codimension 2) into the dense subfield $\theta(\Lambda)$ of $\left(C, v_{r}\right)$. Thus $r=t$ $\left[2\right.$, p. 131]. Since $\left(C, v_{r}\right)$ is complete, this isomorphism has an isometric extension $\psi$ from $\left(C, v_{t}\right)$ on to $\left(C, v_{t}\right)\left[4\right.$, p. 221]. Since $\psi^{-1} \theta(\Lambda)$ has codimension 2 in $C$, and $\theta(\Lambda) \neq \theta(\Phi)$, it follows that $\theta(\Phi)=C$, that is $(\Phi, v) \cong\left(C, v_{t}\right)$.

Conversely suppose that $\Lambda$ has only a finite number of nonisomorphic valued field extensions. If the valuation $v$ on $\Lambda$ is nonarchimedean, it can be extended to any field extension of $\Lambda$ [5, p. 299], and there are an infinite number of nonisomorphic field extensions of any field. Hence $v$ is archimedean, so, by Ostrowski's Theorem [2, p. 131 ], there is an isomorphism $\theta$ from $\Lambda$ on to a dense subfield of $R$ or $C$, and a $t(0<t \leqq 1)$ such that $v=v_{t} \theta$. We identify $\Lambda$ with the subfield $\theta(\Lambda)$ of $C$ with valuation $v_{t}$.

If $B$ is a transcendency basis of $C$ over $\Lambda$, then $\Lambda(B)$, the subfield of $\boldsymbol{C}$ generated by $\boldsymbol{\Lambda}$ and $B$, has only a finite number of nonisomorphic valued extensions, and $C$ is algebraic over $\Lambda(B)$. Now a finite dimensional extension of a valued field has a valuation that extends the given valuation [5, p. 292], so that as an abstract field $\Lambda(B)$ has only a finite number of nonisomorphic finite dimensional extensions. By Proposition $1, \Lambda(B)$ is algebraically closed or $\Lambda(B)$ is real closed with $\Lambda(B)(\sqrt{ }-1)$ algebraically closed; hence $\Lambda(B)=C$ or $\Lambda(B)(\sqrt{ }(-1))$ $=C$. If $B$ is empty the proposition is proved, so we assume that $B$ is not empty. Let $x$ be an element of $B$, and let $\Phi$ be the subfield of 
$C$ generated by $\Lambda, B \backslash\{x\}$, and $\sqrt{ }(-1)(\sqrt{ }(-1)$ may already be in $\Lambda$ ). Then $C=\Phi(x)$ is a simple transcendental extension of $\Phi$. Since $C$ is algebraically closed there is a $\lambda=p(x) / q(x)$, where $p$ and $q$ are polynomials with coefficients in $\Phi$, such that $\lambda^{2}-x=0$. Hence $p(x)^{2}-x q(x)^{2}=0$, so that, by the unique factorization of polynomials in a transcendental element, $x$ is algebraic over $\Phi$. This contradiction completes the proof.

3 Remarks. (i) There are archimedean real closed fields $\Lambda$ contained in $C$ with $\Lambda(\sqrt{ }(-1))=C$ and $\Lambda \cong R$ as a field but not as a valued field. For, it is well known that there are infinitely many field automorphisms $\theta$ of $C$ not leaving $R$ invariant [4, p. 157, say], and $\Lambda=\theta(R)$ is a real field with $\Lambda(\sqrt{ }(-1))=C$. If $\Lambda \neq R$ it cannot be topologically isomorphic to $R$, since the only closed topological subfields of $C$ are $R$ and $C[1, \S 3]$.

(ii) Since the only complete subfields of $\left(\boldsymbol{C}, v_{t}\right)$ are $\boldsymbol{C}$ and $\boldsymbol{R}$, Proposition 2 implies that the only complete valued fields with only a finite number of nonisomorphic valued field extensions are essentially $\left(\boldsymbol{C}, v_{t}\right)$ and $\left(\boldsymbol{R}, v_{t}\right)(0<t \leqq 1)$.

(iii) Another corollary is that a valued field has no proper valued field extension if, and only if, it is isomorphic to $\left(C, v_{t}\right)$ for some $t(0<t \leqq 1)$.

(iv) This last result shows that the only valued field over which the whole Gelfand theory of commutative Banach algebras can be developed is the complex field (see, for example, [6], [7]).

(v) By the Artin-Schreier Theorem [4, p. 316], every proper subfield $\Lambda$ of finite codimension in $C$ is real closed with $\Lambda(\sqrt{ }(-1))=C$. With reference to remark (i) above, it would be interesting to know whether or not every subfield of codimension 2 in $C$ is field isomorphic to $R$.

(vi) In the second half of the proof of Proposition 2, after identifying $\Lambda$ with a subfield of $C$, one may show that the transcendency degree of $\boldsymbol{C}$ over $\boldsymbol{\Lambda}$ is finite, and hence that $\boldsymbol{C}$ is the extension of $\boldsymbol{\Lambda}$ by a finite number of elements. Then a result of $E$. Fried [3] implies that $\Lambda=C$ or $\Lambda$ is real closed with $\Lambda(\sqrt{ }(-1))=C$.

\section{REFERENCES}

1. R. Baer and H. Hasse, Zusammenhung und Dimension topologischer Körperräume, J. Reine Angew. Math. 167 (1932), 40-45.

2. N. Bourbaki, Algèbre commutative. Chaps. 5, 6, Actualités Sci. Indust., no. 1308, Hermann, Paris, 1964. MR 33 \#2660.

3. E. Fried, Algebraically closed fields as finite extensions, Mat. Lapok 7 (1956), 4760. MR $20 \# 6413$. 
4. N. Jacobson, Lectures in abstract algebra. Vol III. Theory of fields and Galois theory, Van Nostrand, Princeton, N.J., 1964. MR 30 \#3087.

5. S. Lang, Algebra, Addison-Wesley, Reading, Mass., 1965. MR 33 \#5416.

6. L. Narici, On nonarchimedean Banach algebras, Arch. Math. (Basel) 19 (1968), 428-435. MR 38 \#5006.

7. N. Shilkert, Non-archimedean Gelfand theory, Pacific J. Math. 32 (1970), 541550.

University of the WitwatersRand, JohanNeSburg, South Africa 